\title{
Experimental Determination of the Superconducting Pairing State in YBCO from the Phase Coherence of YBCO-Pb de SQUIDs
}

\author{
D. A. Wollman, D. J. Van Harlingen, W. C. Lee, D. M. Ginsberg, and A. J. Leggett \\ Department of Physics and Materials Research Laboratory, University of Illinois at Urbana-Champaign, Urbana, Illinois 61801
}

(Received 18 June 1993)

\begin{abstract}
We present an experiment designed to determine directly the symmetry of the pairing state in the cuprate superconductors. From the magnetic flux modulation of YBCO-Pb dc SQUIDs, we determine the spatial anisotropy of the phase of the order parameter in single crystals of YBCO. The experimental results are complicated by SQUID asymmetries and the trapping of magnetic vortices, but taken as a whole give rather strong evidence for a phase shift of $\pi$ that is predicted for the $d_{x^{2}-y^{2}}$ pairing state. This is further corroborated by single junction modulation measurements.
\end{abstract}

PACS numbers: $74.50 .+\mathrm{r}, 74.20 . \mathrm{Mn}, 74.72 . \mathrm{Bk}$

Since the discovery of the high temperature cuprate superconductors, much effort has concentrated on the determination of the superconducting pairing mechanism and in particular the symmetry of the pairing state. Although many symmetries are allowed in principle [1], there is strong experimental evidence that the spin pairing is singlet [2], suggesting an $s$-wave or $d$-wave state. There are also indications that spin fluctuations, thought to be important in the normal state, may also be responsible for the superconductivity. It has been shown within the framework of a Hubbard model [3] and in a phenomenological theory [4] based on NMR measurements [2] that the exchange of antiferromagnetic spin fluctuations could, with suitable assumptions, give rise to pairing in a $d_{x^{2}-y^{2}}$ state with transition temperatures on the order of those observed in $\mathrm{YBa}_{2} \mathrm{Cu}_{3} \mathrm{O}_{7-\delta}(\mathrm{YBCO})$. Measurements of the specific heat [5], thermal conductivity [6], Raman background spectra [7], penetration depth [8], and microwave surface resistance [9] are consistent with the nodes in the energy gap present in the $d_{x^{2}-y^{2}}$ state in that they show evidence for enhanced excitation densities at low temperatures. Recent photoemission experiments [10] give direct evidence for an anisotropic energy gap. All of these measurements, however, are also consistent with an anisotropic $s$-wave state recently proposed [11]. An $s+i d$ state, a superposition of $s$ - and $d$-wave states, is also allowed by symmetry [12].

In this Letter, we present measurements of the phase coherence in bimetallic dc SQUIDs and tunnel junctions made from single crystals of $\mathrm{YBa}_{2} \mathrm{Cu}_{3} \mathrm{O}_{6.8}$ and thin films of the conventional $s$-wave superconductor $\mathrm{Pb}$. This experiment is capable of determining unambiguously the symmetry of the pairing state in YBCO. In particular, it is sensitive to the relative phase of the superconducting order parameter in orthogonal $k$ space directions and thus can distinguish the proposed $d$-wave state from an anisotropic $s$-wave state. Our preliminary results are not conclusive, but taken as a whole rather strongly suggest a state with phase anisotropy consistent with the $d_{x^{2}-y^{2}}$ state.

The basic idea for the experiment was first proposed by Geshkenbein and Larkin [13] as a test for axial $p$-wave pairing symmetry in the heavy fermion superconductors. More recently, Sigrist and Rice [14] arrived at the same scheme by considering anomalous flux entry into $\mathrm{Bi}_{2} \mathrm{Sr}_{2} \mathrm{CaCu}_{2} \mathrm{O}_{8}$ in the paramagnetic Meissner effect [15]. The plan of the experiment is shown in Fig. 1(a). Josephson tunnel junctions fabricated on the orthogonal $a$ and $b$ faces of a YBCO single crystal are connected by a conventional $s$-wave superconductor to form a dc SQUID interferometer. Since the tunneling probability is strongly peaked for electron wave vectors perpendicular to the junction face, the Josephson supercurrent in each junction depends on the phase of the YBCO order parameter orthogonal to the junction. Maintaining phase coherence around the SQUID loop forces the constraint $\phi_{a}$ $-\phi_{b}+2 \pi \Phi / \Phi_{0}+\delta_{a b}=0$ on $\phi_{a}$ and $\phi_{b}$, the gauge-invari-

(a)

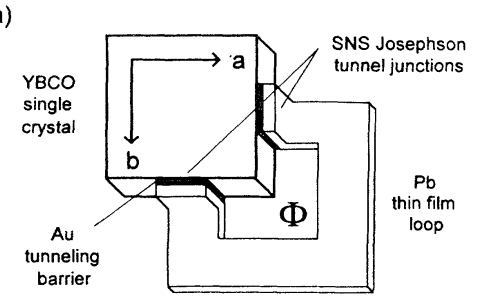

(b)

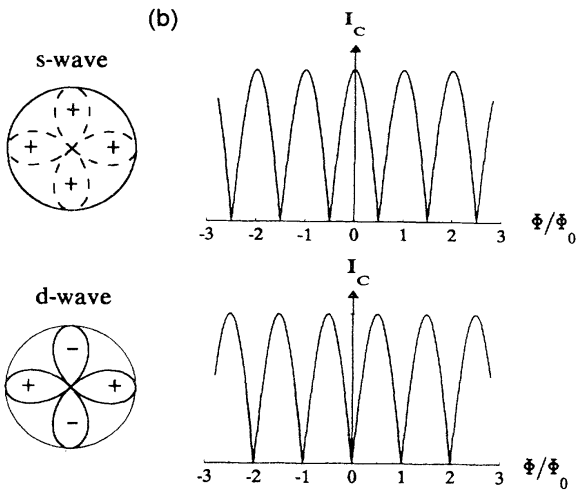

FIG. 1. (a) Configuration of YBCO-Pb corner dc SQUIDs. (b) Predicted modulation of the SQUID critical current vs applied magnetic flux for the $s$-wave and $d_{x^{2}-y^{2}}$ pairing states. 
ant phase differences across the junctions on the $a$ and $b$ faces of the crystal. Here, $\Phi=\Phi_{\text {ext }}+L J$ is the magnetic flux in the loop which includes externally applied flux $\Phi_{\text {ext }}$ and flux from the circulating current $J$ around the SQUID loop with self-inductance $L$. The extra term $\delta_{a b}$ is the intrinsic phase shift inside the YBCO crystal between pairs tunneling into the crystal in the $a$ and $b$ directions. For a symmetric de SQUID with junction critical currents $I_{0}$ in the limit of zero inductance, the maximum supercurrent modulates with applied flux according to $I_{c}=2 I_{0}\left|\cos \left(\pi \Phi_{\text {ext }} / \Phi_{0}+\delta_{a b}\right)\right|$. For finite values of the screening parameter $\beta=2 L I_{0} / \Phi_{0}$, the modulation depth of the critical current is reduced. For large $\beta$, $\Delta I_{c} / I_{c} \sim 1 / \beta$.

The two principal cases are indicated in Fig. 1(b). If the YBCO has $s$-wave symmetry (isotropic or not), the phase of the order parameter is the same at each junction inside the YBCO so that $\delta_{a b}=0$ and the circuit will behave as an ordinary dc SQUID. In particular, the critical current is a maximum for zero applied field as indicated. In contrast, for $d_{x^{2}-y^{2}}$ symmetry the order parameter has the form $\Delta\left(k_{x}, k_{y}\right)=\Delta_{0}\left[\cos \left(k_{x} a\right)-\cos \left(k_{y} a\right)\right]$, featuring an intrinsic phase shift $\delta_{a b}=\pi$ between the $a$ and $b$ directions as shown in the polar graph. At zero flux, the junctions are out of phase and a circulating current flows to maintain phase coherence around the SQUID loop. As a result, the critical current will instead be a minimum at zero flux. If the pairing state has $s+i d$ symmetry, the phase shift can take on any value; for an equal admixture of the $s$ and $d$ components, $\delta_{a b}=\pi / 2$. Thus, by observing the modulation of the SQUID response vs flux, it is possible to deduce the symmetry of the pairing state of the YBCO sample. The elegance of the experiment is that it depends only on the relative phases of the order parameter and is not sensitive to the type, size, or critical currents of the constituent junctions.

High-quality YBCO single crystals obtained by a fluxmelt technique [16] are used for the experiment. Typical dimensions are $0.50 \mathrm{~mm} \times 0.75 \mathrm{~mm}$ in the $a-b$ plane with thicknesses of 20-30 $\mu \mathrm{m}$. Both twinned and untwinned crystals have been tested and give the same results. Only crystals with smooth, flat natural growth faces and a sharp corner are selected. Samples are prepared by first thermally evaporating $100-200 \mathrm{~nm}$ of $\mathrm{Au}$, masked into sections approximately $75 \mu \mathrm{m}$ wide, onto the $a-c$ and $b-c$ faces of the crystals. The gold is annealed at $400^{\circ} \mathrm{C}$ for several hours to produce low resistance contacts. The crystal is then placed with the $c$ axis vertical onto a small droplet of polyamide on a glass slide; the polyamide adheres to the bottom face of the crystal, providing a smooth slope from the substrate up to the edges. Finally, a $\mathrm{Pb}$ film $(800 \mathrm{~nm})$ is deposited through a photoresist mask to make contact to the Au-coated edges, define the SQUID loop, and form electrical leads. The dimensions of the SQUID loops vary between samples; a typical size is $50 \mu \mathrm{m} \times 50 \mu \mathrm{m}$. Besides the corner SQUID loop connecting junctions on the $a$ and $b$ faces, several SQUIDs
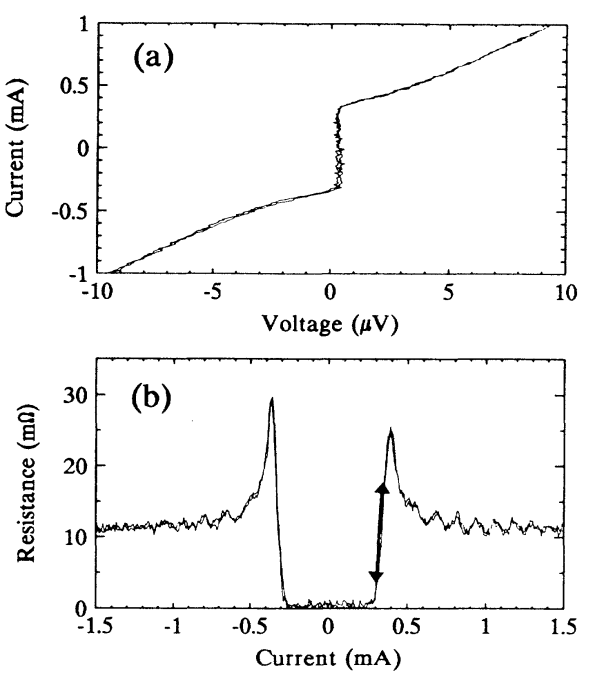

FIG. 2. (a) Current vs voltage characteristics of a $\mathrm{YBCO}-\mathrm{Pb}$ SQUID with a critical current of $300 \mu \mathrm{A}$. (b) Dynamic resistance vs current for the same SQUID. The arrows indicate the bias range for which flux modulation data are taken.

are also fabricated on each crystal with both junctions on the same face-these edge SQUIDs serve as control samples since both junctions sample the same $k$-space direction. For all measurements, the SQUIDs are zero field cooled inside a mu-metal shield; we estimate the ambient field to be less than $1 \mathrm{mG}$.

The YBCO-Au-Pb tunnel junctions formed by this method are SNS (superconductor-normal metal-superconductor) junctions. The critical currents depend sensitively on the Au thickness and annealing, and are strongly temperature dependent. At $3 \mathrm{~K}$, where most of the data presented here were taken, values range from 10-500 $\mu \mathrm{A}$. The junctions exhibit nearly ideal RSJ (resistively shunted junction) current-voltage characteristics. Figure 2(a) shows the current vs voltage curve for a YBCO-Pb SQUID with a total critical current of 300 $\mu \mathrm{A}$. The critical current modulates with flux by only $1 \%-2 \%$ in this sample, implying $\beta \sim 50-100$. The modulation is most easily detected by biasing the SQUID at a constant current and measuring the dynamic resistance vs flux. For all measurements reported here, the SQUIDs are biased in the noise-rounded region below the resistance maximum, as indicated in Fig. 2(b), where we expect that an increase in the critical current will correspond to a decrease in the resistance. In samples with low critical currents, the noise-rounded region can extend all the way to zero bias current.

The resistance vs applied flux for a YBCO-Pb SQUID with a thermodynamic critical current of about $50 \mu \mathrm{A}$ is shown in Fig. 3 for several values of bias current. Besides the expected periodic modulation, there is a currentdependent phase shift. This shift is caused by the asymmetric division of the bias current through the two paral- 


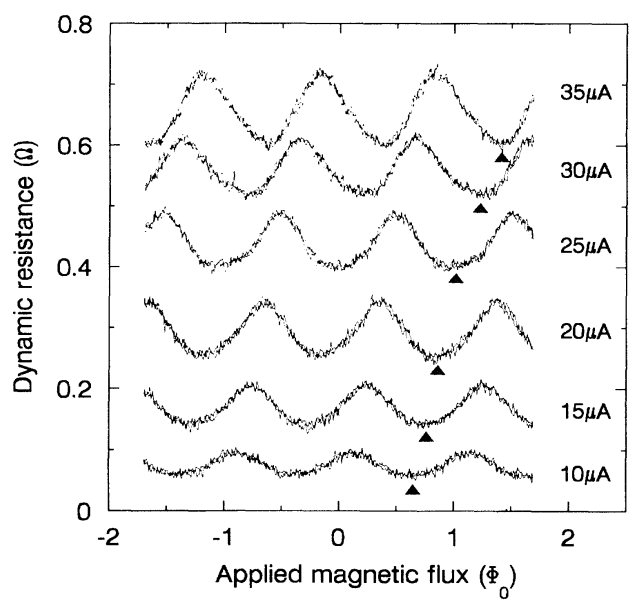

FIG. 3. Modulation of the dynamic resistance vs applied magnetic flux for different bias currents in a corner SQUID with a thermodynamic critical current of about $50 \mu \mathrm{A}$. Because of noise rounding, the modulation can be measured for currents well below the critical current.

lel branches of the SQUID which generates a net flux in the SQUID loop. Asymmetries in both the critical currents of the junctions and the inductances of the branches contribute to this effect. Consider an asymmetric SQUID with junction critical currents $I_{1}=(1+\alpha) I_{0}$ and $I_{2}=(1-\alpha) I_{0}$ and branch inductances $L_{1}=(1+\eta) L / 2$ and $L_{2}=(1-\eta) L / 2$, where $\alpha=\left(I_{1}\right.$ $\left.-I_{2}\right) / 2 I_{0}$ is the fractional critical current asymmetry and $\eta=\left(L_{1}-L_{2}\right) / L$ is the corresponding inductance asymmetry. Near the critical current, the flux $\Phi$ linking the SQUID loop varies linearly with the bias current $I$, approximately as $\Phi=\frac{1}{2}(\alpha+\eta) L I=\frac{1}{2}(\alpha+\eta) \beta\left(I / 2 I_{0}\right) \Phi_{0}$. The asymmetry is also responsible for the resistance oscillations visible in Fig. 2(b).

To extract the phase shift $\delta_{a b}$ inside the YBCO crystal, we must determine the phase of the $R$ vs $\Phi$ curves at zero bias current. This is done by plotting the value of applied flux at which the resistance is a minimum (corresponding to a maximum in the critical current) vs the bias current and extrapolating to zero current, as shown in Fig. 4 for several SQUIDs. We can determine the zero bias flux intercept to an accuracy of $\pm 0.1 \Phi_{0}$, depending on the asymmetry of the SQUID which controls the slope, and on the critical current which controls the extent of the extrapolation. For a corner SQUID, $s$-wave pairing gives an intercept at $\Phi=0$; the $d_{x^{2}-y^{2}}$ state would yield an intercept at $\Phi=\Phi_{0} / 2$. In Fig. 4(a), we show measurements and linear extrapolations for seven corner SQUIDs, each cooled slowly in zero field. The intercepts vary from $(0.3-0.6) \Phi_{0}$. Thus, although neither the $s$-wave nor the $d$-wave signature is strictly indicated, each SQUID does exhibit a significant phase shift of order $\pi$ consistent with a $d_{x^{2}-y^{2}}$ state.

The variation in the intercept is likely due to trapping of magnetic flux vortices. Flux trapped near one of the
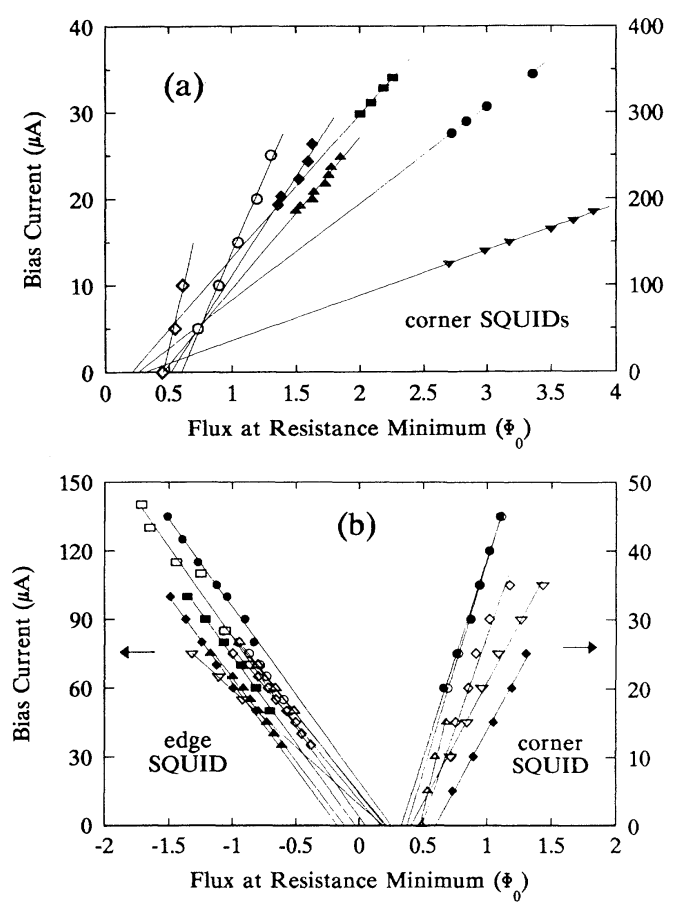

FIG. 4. (a) Extrapolation of the measured SQUID resistance minimum vs flux to zero bias current for seven corner SQUIDs. The filled symbols are scaled to the right axis. (b) Extrapolations for a corner SQUID and an edge SQUID on the same crystal. Each curve represents a different cooldown of the sample.

Josephson junctions can reduce its critical current and modify the SQUID asymmetry, causing a change in the slope of the current-dependent phase shift. A vortex can also be trapped near the SQUID loop, linking flux to the SQUID and inducing a parallel shift in the curves. This is demonstrated in Fig. 4(b) which shows data from a corner SQUID and an edge SQUID with comparable loop areas fabricated on the same crystal. Each curve is from a cooldown of the sample after warming to above the transition temperature of YBCO. The corner SQUID intercepts cluster around $\Phi_{0} / 2$, suggesting a statistical distribution of trapped configurations about the intrinsic (zero trapped flux) result. In support of this are the data from the edge SQUID for which the intercepts distribute about 0 , as is expected independent of the pairing state.

An alternative method of determining the pairing state is to study the magnetic flux dependence of the critical current of a single Josephson junction. For a uniform junction, the critical current has the usual Fraunhofer diffraction pattern form $I_{c}(\Phi)=I_{0} \mid \sin \left(\pi \Phi / \Phi_{0}\right) /(\pi \Phi /$ $\left.\Phi_{0}\right) \mid$, as shown in Fig. 5(a). This should be observed for a YBCO-Pb junction on the edge of the crystal independent of the pairing state. If, however, a junction is formed on the corner of the crystal so that part of the tunneling is in the $a$ direction and part in the $b$ direction, the critical current modulation reflects the pairing state 

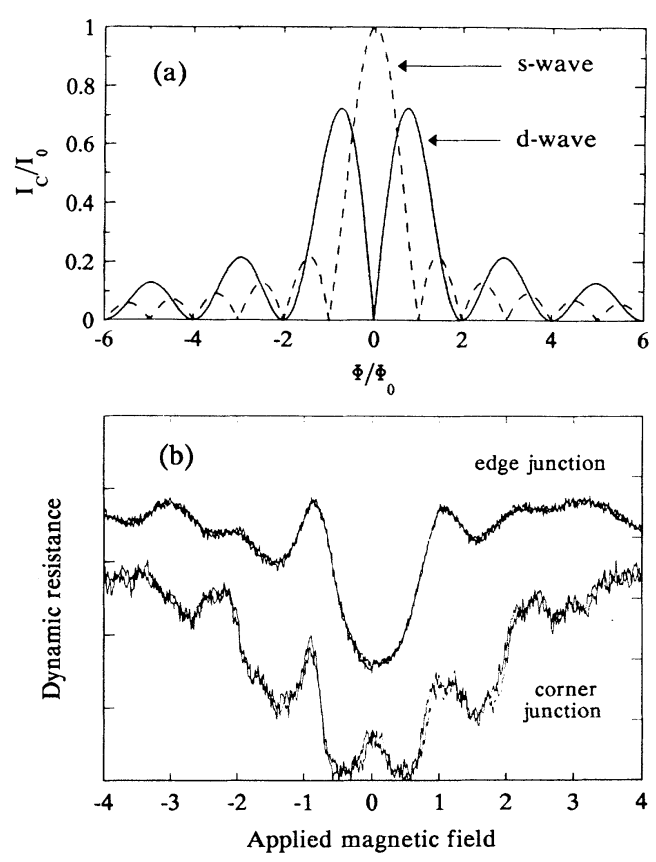

FIG. 5. (a) Calculated critical current vs flux for a YBCO$\mathrm{Pb}$ single corner junction for $s$-wave and $d$-wave symmetry. (b) Measured resistance vs flux for a corner junction and a single junction on one edge (shifted vertically for clarity).

symmetry. For $s$-wave pairing the Fraunhofer pattern is again obtained; for $d$-wave pairing the $\pi$ phase shift between orthogonal directions causes a cancellation of the current into the two faces at zero flux and results in a modified pattern. For equal $a$ and $b$ components, the functional form is $I_{c}(\Phi)=I_{0}\left|\sin ^{2}\left(\pi \Phi / 2 \Phi_{0}\right) /\left(\pi \Phi / \Phi_{0}\right)\right|$, as shown in Fig. 5(a). To test this, we have fabricated single junctions on the edge and corner of a YBCO crystal. The resistance vs applied flux is shown in Fig. 5(b); the external field is applied along the $c$ axis so that it threads the junction barrier. The edge junction modulation is consistent with a Fraunhofer diffraction pattern, in particular exhibiting a minimum in the resistance at zero flux. In contrast, the corner junction has a maximum in the resistance at zero flux, as would be expected for the $d$-wave state. Although we have only studied the single junction modulation in this one pair of junctions, this result is compelling since we expect it to be less sensitive to asymmetry and trapped flux than the SQUID measurements.

In conclusion, we have measured the phase shift of the order parameter between the orthogonal $a$ and $b$ directions in YBCO crystals by studying the phase coherence of $\mathrm{YBCO}-\mathrm{Pb} \mathrm{dc}$ SQUIDs. Accounting for junction asymmetries and trapped magnetic flux, the data collectively give evidence for a phase shift of order $\pi$, consistent with the $d_{x^{2}-y^{2}}$ pairing state. Preliminary single junction diffraction measurements also support this symmetry.
Further measurements are underway to verify these results and to test their dependence on crystal twinning, sample geometry, and the orthorhombic structure of YBCO. In conjunction with complementary measurements of the anisotropy of the magnitude of the order parameter, this experiment should allow an unambiguous determination of the superconducting pairing state of the high temperature cuprates.

We are grateful for motivation and insight provided by D. Pines, C. P. Slichter, N. D. Goldenfeld, M. B. Salamon, M. V. Klein, and I. Kosztin, and for technical advice from J. P. Rice in the early stages of this experiment. This experiment was supported by the National Science Foundation and the Frederick Seitz Materials Research Laboratory under Grant No. NSF DMR 8920538. We acknowledge supplementary support from the NSF Science and Technology Center for Superconductivity under Grant No. NSF DMR 91-20000 (W.C.L., A.J.L.); one of us (D.A.W.) is supported by an NSF Graduate Research Fellowship. We also acknowledge extensive use of the facilities of the MRL Microfabrication Laboratory.

[1] J. F. Annett, N. Goldenfeld, and S. R. Renn, Physical Properties of High Temperature Superconductors II, edited by D. M. Ginsberg (World Scientific, Teaneck, NJ, 1991).

[2] S. E. Barrett et al., Phys. Rev. Lett. 66, 108 (1991); J. A. Martindale et al., Phys. Rev. B 47, 9155 (1993).

[3] N. E. Bickers, D. J. Scalapino, and S. R. White, Phys. Rev. Lett. 62, 961 (1989).

[4] P. Monthoux, A. Balatsky, and D. Pines, Phys. Rev. B 46, 14803 (1992).

[5] S. E. Stupp and D. M. Ginsberg, Physical Properties of High Temperature Superconductors III, edited by D. M. Ginsberg (World Scientific, Teaneck, NJ, 1991).

[6] R. C. Yu, M. B. Salamon, and W. C. Lee, Phys. Rev. Lett. 69, 1431 (1992).

[7] S. L. Cooper and M. V. Klein, Comments Condens. Matter Phys. 15, 99 (1990).

[8] J. F. Annett, N. Goldenfeld, and S. R. Renn, Phys. Rev. B 43, 2778 (1991); W. N. Hardy et al., Phys. Rev. Lett. 70, 3999 (1993).

[9] D. A. Bonn et al., Phys. Rev. B 47, 11314 (1993).

[10] Z.-X. Shen et al., Phys. Rev. Lett. 70, 1553 (1993).

[11] S. Chakravarty, A. Sudbo, P. W. Anderson, and S. Strong, Science 261, 337 (1993).

[12] G. Kotliar, Phys. Rev. B 37, 3664 (1988).

[13] V. B. Geshkenbein and A. I. Larkin, Pis'ma Zh. Eksp. Teor. Fiz. 43, 306 (1986) [JETP Lett. 43, 395 (1986)]; V. B. Geshkenbein, A. I. Larkin, and A. Barone, Phys. Rev. B 36, 235 (1987).

[14] M. Sigrist and T. M. Rice, J. Phys. Soc. Jpn. 61, 4283 (1992).

[15] W. Braunisch et al., Phys. Rev. Lett. 68, 1908 (1992).

[16] J. P. Rice et al., Phys. Rev. B 41, 6532 (1990). 